\title{
Wikipedia Edit-a-thons: Thinking Beyond the Warm Fuzzies
}

\author{
Danielle Robichaud \\ Digital Archivist \\ University of Waterloo
}

\begin{abstract}
Wikipedia edit-a-thons represent a unique and fruitful avenue for galleries, libraries, archives and museums (GLAMs) to engage with new and existing segments of their user communities. Encompassing information and technical literacy skills development, the success of edit-a-thon campaigns, such the annual art+feminism events, demonstrate their utility and value as outreach initiatives. What is less clear are the logistical, ethical and professional development implications of hosting these events. Centering first-hand experience and concrete examples, this paper explores GLAMbased edit-a-thons through a practical and actionable lens. Topics covered include: what is required to successfully host an edit-a-thon; the importance of leading by example in a volunteer-reliant economy; and the transferable work skills gained by hosting and participating in edit-a-thons.
\end{abstract}

\section{Introduction}

I started editing Wikipedia through an archival lens more than two years ago, driven by sheer dismay. At the time I was working at the Henri J.M. Nouwen Archives \& Research Collection on various digital projects. The work included online outreach like adding links to findings aids on our website to relevant Wikipedia pages. Working with Nouwen's personal papers, I knew his life better than most, which made the poorly written page about his career flat-out distressing. Not only did it rely on a plagiarized timeline created by the Archives, it was heavily focused on one aspect of Nouwen's life rather than the spectrum of experiences and contributions that define a person. What started as the means-to-an-end addition of a finding aid link led to months of regular editing to improve the page.

Since that time I have attended and hosted various edit-a-thons, worked with archivist Amanda Hill to develop a Wikipedia for Archivists workshop, served as McMaster University's Wikipedia Visiting Scholar, and talked endlessly about why galleries, libraries, archives, and museums (GLAMs) should be playing a larger role in the Wikipedia landscape. As a result, I get asked about Wikipedia. A lot.

It feels wonderful when it happens and I am always happy to help, but most of these exchanges are fairly standard-people ask general questions about editing or training and I do my best to point them to relevant resources. What gets missed are the lessons that have come my way through trial, error, and poorly-placed good intentions. 
With that in mind, the following maps out some of what goes hand in hand with GLAMs contributing to Wikipedia, such as: What is an edit-a-thon and how much work goes into hosting one? How can cultural professionals lead by example given our problematic reliance on unpaid, volunteer labour? How do you properly prepare new editors to confidently contribute to Wikipedia? And, finally, how does Wikipedia align with professional development goals in a climate where less is more?

\section{What is an Edit-a-thon?}

Wikipedia edit-a-thons are planned events aimed at improving or creating Wikipedia pages. Often tied to an overarching theme, anniversary, or collection, they are particularly useful for providing new editors with a structured and supportive space where they can edit and ask questions while doing so. For cultural and heritage organizations, edit-a-thons have the added benefit of being a great outreach and engagement initiative. In addition to the more immediate outcome of collectively improving the internet's most popular information resource (LaFrance, 2016), edit-athons are an opportunity for community members to get familiar with your holdings, your services, and the expertise of you and your colleagues.

If you work at a GLAM and want to host an edit-a-thon, I encourage you to familiarize yourself with these two resources:

1. Wikipedia's How to run an edit-a-thon guide ${ }^{1}$ is a comprehensive overview of the practical and logistical considerations required to successfully run this type of event. It is the best place to find a realistic step-by-step description of how an edit-a-thon comes together and what you can expect while planning and hosting.

2. The Wikipedia Library's Best practices for Librarians, Archivists and Cultural Professionals who want to link to collections on Wikipedia does a great job of situating GLAM organizations in the Wikipedia landscape. It includes an explanation about handling conflicts of interest and provides a series of entrylevel contribution examples for GLAMs looking to improve the discovery of their research collections.

Beyond these examples you will find that there is a wealth of 'how to' documentation available both on and off Wikipedia, often resulting in the same instructions being written multiple ways, for multiple audiences, and in various formats. This means there is no need to reinvent the wheel: instead, focus on finding the right fit for your organization and edit-a-thon participants. If a page is too long, there is very likely a summary available. If you prefer visuals over text, walk-throughs with screenshots and videos are common. Work through the available guides and tutorials and make note of what you find useful as you go. The list of resources you pull together may very well end up being the useful links you provide to participants during your first edit-a-thon.

\footnotetext{
${ }^{1}$ Editor's note: Because of the large number of citations directly to Wikipedia in this article, Partnership has decided to forego the usual academic citation formatting, and instead simply provide in-text links.
} 


\section{Lead from Experience}

A common concern I hear from people curious about editing Wikipedia, but reluctant to take the plunge, is that they don't believe they know enough about the site or a particular topic to contribute. The sentiment mirrors the research findings of Julia B. Bear and Benjamin Collier (2016), who found that lack of confidence and avoidance of potential conflict impact how women interact with the platform. With that in mind, if you are considering running an edit-a-thon and have never edited Wikipedia, it is important that you hit pause on the event planning and get editing. ${ }^{2}$ Not only will it allow you to be a more informed and supportive host, personal experience allows for the provision of concrete examples that bring potentially daunting guidelines to life in a relatable manner.

Though you will undoubtedly find your own rhythm for hosting an edit-a-thon, as both participant and instructor, I have found that personal anecdotes are a reliable counterpoint to information overload. Each time I talk about my involvement with Wikipedia I make sure to discuss how I have handled screwing up and how I have learned new tasks. I find that being candid about the hiccups I encounter while editing humanizes the experience and demonstrates that what may feel like a rocky learning curve is normal and ongoing, even for the people leading the event. If, for example, a participant is struggling with task X, there is immense value in being able to share how I dealt with the same issue or figuring out the answer together. Here's an example any new editor is going to struggle with:

\section{How do I deal with the frustration, embarrassment, or hurt feelings that arise when contributions are reverted or my pages deleted?}

I do my best to assume good faith and look at what happened as a learning opportunity. I once added a singer-songwriter's Twitter account to the external links section of their page only to have the edit immediately reverted (see Jim Guthrie (singer-songwriter): Difference between revisions). It stung, but the result was learning about the External links guidelines, which proved useful as I started undertaking more extensive page revisions. For example, I learned that web links should not be added to the External links section if they have been used as references in the main body of the page.

Of course not every exchange is going to be quite as useful. Another time I added a citation needed tag to unreferenced information. A seasoned editor responded by adding a link and leaving the comment: "Why whine when it's easy to find the reference?" 3 In that instance I learned that some editors are jerks and moved on.

Wikipedia is ultimately about making knowledge creation available to everyone. The site's emphasis on policies and guidelines rather than hard-and-fast rules means that

\footnotetext{
${ }^{2}$ Although there are a number of tutorials available, Wikipedia's step-by-step tutorial or the Wikipedia Adventure, a gamified training model, are good places to start.

${ }^{3}$ I am intentionally leaving this exchange uncited. Poor editor behaviour doesn't need more attention than it already gets.
} 
there isn't one right way to contribute. Discussing and working through shared challenges as a group is a good way to model technical problem solving and empower new editors to find solutions that work for them. ${ }^{4}$

\section{Lead by Example}

Though some will undoubtedly argue the point, there are instances in the GLAM community where interest in technology skills and related events are driven by one of two things: lack of resources, and the fear of missing out on trendy outreach initiatives. In my experience, Wikipedia edit-a-thons check both of those boxes. Many GLAM organizations lack the resources to undertake online outreach efforts beyond maintaining a basic website or social media presence, making further promotion difficult. This makes edit-a-thons doubly appealing: people use your holdings to improve or create pages that benefit your organization during a feel-good event, and they do it for free. While I am the first to admit that it is, indeed, a divine exchange, I must also point out that this needs to be a two-way street.

If we as GLAM professionals are going to invite people to use our resources to create Wikipedia content, it is important that we do so while acknowledging that we have a history of relying on un(der)paid and crowdsourced labour to bring online content to life. ${ }^{5}$ Counter to conflict of interest guidelines, which outline why people and organizations should refrain from editing their own pages, many GLAMs continue to see Wikipedia as a tool for self-promotion. There is a persistent and narrow focus on embedding finding-aid links in relevant pages and creating dedicated pages for our organizations. While understandable, given the output-driven metrics our funding often relies on, it is an approach that is short-sighted and unfair to the people we are inviting to edit-a-thons. An easy remedy to this practice is to lead by example and contribute content, ourselves, that goes beyond what is immediately beneficial to our organization.

Wikipedia is an open encyclopedia, not a promotional website. If your organization already has a website, a Wikipedia page (restating what has already been captured on your site) is of no added benefit to your patrons or Wikipedia readers. What, instead, greatly benefits readers is the subject expertise of you and your colleagues. As GLAM professionals we are well versed in the people, subjects, and events represented in our holdings. This means that we know the best resources about specific people or events to improve existing pages, can easily identify gaps in how a topic is covered, are able to introduce balance by contributing alternative viewpoints, and know what pages or subsections are missing when it comes to addressing the persistent Wikipedia issue of underrepresented people(s) and events. While the lure of a dedicated page about your

\footnotetext{
${ }^{4}$ Remember: sometimes walking away and directing your attention to one of the other millions of pages in need of improvement and your expertise is the best course of action.

${ }^{5}$ Recent pieces by Mayer (2016), Reyes (2014), and Williams (2016) examine the issue of unpaid, uncredited work in the cultural realm, while Howard's (2015) work, regarding labour issues inherent to Wikipedia itself, helps to underscore the importance of a reflexive consideration of how, why and when GLAMs engage with Wikipedia.
} 
organization is hard to resist, and can be difficult to justify, ${ }^{6}$ there is endless value in contributing more substantively by way of page development and improvement, which can be tracked over time to draw attention to your organization's contributions and value. $^{7}$

When we focus time and energy strictly on developing a page about our organization while leaving special topics to everyone else, we are sending the message that Wikipedia is valuable to us only as a promotional tool. That is contrary to the "help improve Wikipedia' rationale for holding edit-a-thons and goes against established editing guidelines. Worse, it fails to take advantage of and showcase the incredible range of research and writing skills cultural professionals make use of everyday. The narrow focus ultimately ensures that our edit-a-thon efforts remain shallow and disingenuous.

Ideally, the goal of an edit-a-thon should be helping participants expand their technology and information literacy skills so that they can confidently edit Wikipedia over time. This goal is undermined when we focus on self-promotion rather than substantial and meaningful contributions. For example, disrupting and diversifying the homogenous Western male lens (Bergen, 2016; Boboltz, 2015; Simonite, 2013; Wagner, GraellsGarrido, Garcia, \& Menczer, 2016; Zandt, 2013) is an effort that has the clear public benefit of reducing the most unappealing and alienating aspects of the site.

\section{Be Prepared}

Once you have familiarized yourself with editing basics, you need to consider how to prepare edit-a-thon attendees for the realities of editing in an open environment. Wikipedia has a reputation of being uninviting to new editors (Torres, 2016). ${ }^{8}$ In light of this climate, I underscore that teaching others how to edit Wikipedia comes with a responsibility to be honest and upfront about what they can expect. For example, a key reason new editors get 'bit' is a lack of understanding about established guidelines. So, yes, while anyone can be bold and start editing, not everyone is going to make edits that stick (McQuigge, 2013). One reason is overzealous page deletion-the removal of a new or in-progress page by another editor due to what is perceived as poor quality writing, page development, topic choice, or supporting references. Although deletion guidelines encourage improving problem pages rather than simply deleting them, it is

\footnotetext{
6 Standalone Wikipedia pages are evaluated based on notability demonstrated by multiple secondary sources that have no direct affiliation with the person or organization of interest. This means that your GLAM's website cannot be solely relied on as a notability resource. Using historical news articles to provide contextual information about the department and major acquisitions that would not normally appear on your institutional website is one way to approach the task. I used a similar method as McMaster's Wikipedia Visiting Scholar when I created a Wikipedia page the William Ready Division of Archives and Research Collections.

7 Two common examples are web traffic tracking, as demonstrated by University of Houston Libraries Digital Services Department (Elder, Westbrook \& Reilly, 2012), and the creation of Wikipedia project pages (Raub, 2015). Wikipedia also provides a variety of statistics including page views and image use frequency that can be used to monitor page and content use over time.

${ }^{8}$ See note 7 above for scholarship regarding Wikipedia editor homogeneity.
} 
not uncommon for pages created shortly after, or sometimes during, edit-a-thons to succumb to swift deletion.

As an edit-a-thon host and instructor part of your job is to ensure that new editors understand the framework of a good page so that their hard work isn't summarily deleted. One way to do so is to pre-select useful editing resources and tutorials for participants to work through on their own. Consider working these into a project page that is made available prior to the event so that participants can come with specific questions in hand and know where to find what they need while editing. I encourage you to also leave the project page in place long-term so that participants have something familiar to refer back to after the event. The benefits of putting a project reference page in place are myriad:

- Cherry-picking what you think are the most appropriate instructional resources for your audience minimizes the potential for information overload. It also gives everyone-GLAM staff and edit-a-thon participants alike-a common set of reference points. The page can be hosted on Wikipedia itself, like the main page used by the Toronto branch of art+feminism, or it can be hosted by your organization, like the LibGuide used by the Pollard Memorial Library and the UMass Lowell Library (Marks, 2016).

- Every edit-a-thon participant will have different learning preferences and requirements. Some will want to work autonomously and others will require more hands-on support and structure. Having a stable resource available allows people to explore on their own before, during, or after the event, while giving everyone the same foundation to work from. It also encourages people to learn and work at their own pace.

- A stable space makes it easier to identify where work needs to be done, which resources can be used to accomplish that work, and how that work has evolved over time. One appealing approach is to create a defined project space where events, suggested improvement tasks, and editing resources can be accessed. An example is the project page used by the Newcomb Archives and Vorhoff Library (Raub, 2015). This is a particularly useful approach for organizations seeking to more formally track and report on their contributions over time using Wikipedia tools.

- A reliable project page makes your event more accessible by facilitating participation by people unable to attend in person (including those in other regions!). It also allows editors who participated to refer back to the list of resources and suggested improvements long after the event has ended.

\section{How is Wikipedia Professional Development and how do I Make the Case for Contributing at my Place of Work?}

Depending on your employment environment, you may be unable to contribute as part of your day-to-day professional activities, but there is still a lot to learn from the comfort 
of your own couch and there is no expensive registration fee for doing so. Plus, the benefits of editing Wikipedia accrue whether the editing takes place at home or at work.

\section{Developing Plain English writing skills}

The key to developing a good Wikipedia article is writing in a manner that is "clear, precise and relevant to the reader." This includes mastering a neutral point of view and an ability to summarize information in an easy to understand manner. In the professional realm this approach is more commonly referred to as writing in plain English, and is a foundational aspect of (web) accessibility (Turner, 2016; ARL, 2014). Familiarizing yourself with Wikipedia writing guidelines and putting them to use while editing is a good way to improve how effectively you communicate with audiences of all kinds.

\section{Instructional support}

Wikipedia is increasingly used as part of assigned academic coursework and GLAM training initiatives, a trend reflected by the Wiki Education Foundation's development of Instructor-specific resources (WikiEdu, n.d.). If you are in, or wish to land, an educational outreach or liaison role, having a baseline understanding of Wikipedia guidelines and editing practices will allow you to play a key role in student and instructor support. It will also allow you to make recommendations about underrepresented areas of interest that can be expanded by using your organization's special collections holdings or scholarly resources.

\section{Information Literacy Skills}

Wikipedia is an excellent tool for information literacy instruction. We already know that Wikipedia is the most used information resource online, but whether readers know how to evaluate what they find when they get there is another story. Understanding how to review a page's history for clues about writing development and intent, assess the referenced citations for reliability, verify the accuracy of presented information, and make minor edits to page content information is to your advantage as an instructor, reference desk employee, or support team member. These are the types of skills required in the digital age, and using an existing Wikipedia page is a good way to demonstrate them during instruction workshops and outreach.

\section{Project Management}

From promotions and outreach to on-site logistics and coordination, hosting an edit-athon is a good way to hone your project management skills. The flexibility of the size, format, and focus of the events means that you can start small and expand over time as you learn what works for your audience and colleagues.

\section{Public Speaking Skills}

Being able to learn a new platform and provide a high-level overview to others is an important skill that gets rusty from disuse. Given how much there is to know about 
editing Wikipedia, teaching others about the site and how to contribute is a great way to work on determining what information is mission critical and what information can be learned as the need arises.

\section{What Else do you Need to Know?}

- Be ready and willing to admit when you don't know the answer. I have been editing actively for well over two years and learn something new on a regular basis. Confessing that you don't know the answer is an opportunity to demonstrate that not knowing how to do something is a normal part of the Wikipedia learning process. Additionally, it opens the door for modelling how to find the answer or, better yet, to learn how to do it from another edit-a-thon participant. Reciprocal learning is a beautiful thing that shifts the power dynamic from a one-way exchange to a community-driven project.

- Consider making edit-a-thons a regular drop-in event so that participants can develop their skills over time and access on-site or subscription-based resources they may otherwise be unable to use. Some institutions, including the Art Gallery of Ontario, have found that the community preferred regular opportunities to meet with other editors and shifted to quarterly rather than annual art+feminism events (Mohyeddin, 2016). The good news is that these don't have to be resource intensive. Over time regular participants and on-site staff will develop the skills to support each other, minimizing required resources beyond ensuring that a dedicated work space, relevant on-site resources, and dedicated staff support are available.

- Wikipedia editors work for free and have developed their knowledge over time by trial and error. They are not obliged to help or make the learning process easier for you, although many do (enthusiastically!) without prompting. Always be mindful that there is a difference between asking for clarification based on your reading of training resources and asking someone to do the work for you. If you make use of someone's time and energy, acknowledge and thank them for it.

- Make use of the Article Wizard to minimize the risk of having articles by new editors deleted. The feature creates articles in a Draft namespace where everything is understood to be a work in progress. Articles can be submitted for review and reworked until they are ready to be moved to the main Wikipedia environment.

- Representation matters. Giving people the tools to participate in the creation of shared knowledge on a free and openly available platform makes room for voices and perspectives that are traditionally excluded from descriptive records, finding aids, and exhibits institutionally managed by GLAMs. ${ }^{9}$ Helping people

\footnotetext{
${ }^{9}$ There is an extensive body of scholarly literature that has examined the implications of excluding diverse, community-based input from the management and description of cultural heritage items. Many authors have examined these issues while putting forward actionable suggestions for disrupting this
} 
understand how to evaluate and contribute to the development of Wikipedia content is a meaningful step toward diversifying, decolonizing, and improving community participation in cultural heritage work.

Love it or hate it, when it comes to information seeking behaviour, Wikipedia is a go-to resource for many. Learning how to edit Wikipedia pages and helping others to do the same is an important part of acknowledging that despite our best efforts (and extensive website redesigns) we often have to go to where people are to facilitate access to accurate and reliable information. Edit-a-thons are a high-impact approach to fostering community engagement, online resource promotion, technical skills development, and information literacy. If you decide to host one, make sure to give yourself, your organization and your participants the support they need to succeed.

\section{References}

Association of Research Libraries. (2014) Web accessibility toolkit.

Bear, J. B., \& Collier, B. (2016). Where are the women in Wikipedia? Understanding the different psychological experiences of men and women in Wikipedia. Sex Roles, 74(5), 254-265.

Bergen, S. (2016). Linking in: How historians are fighting Wikipedia's biases. Perspectives on History.

Boboltz, S. (2015). Editors are trying to fix Wikipedia's gender and racial bias problem. The Huffington Post.

Caswell, M.L. (2014). Seeing yourself in history: Community archives in the fight against symbolic annihilation. The Public Historian. 36(4), 26-37.

Drake, J. (2016). RadTech meets RadArch: Towards a new principle for archives and archival description. On Archivy.

Elder, D., Westbrook, R.N., \& Reilly, M. (2012). Wikipedia lover, not a hater: Harnessing Wikipedia to increase the discoverability of library resources. Journal of Web Librarianship. 6(1), 32-44.

Howard, D. (2015). Labor and the new encyclopedia. DIS magazine.

trend. Examples include: Caswell (2014); Drake (2016); Mills (2016); and Young (2016). The work of the Great Lakes Research Alliance for the Study of Aboriginal Arts and Cultures (GRASAC, n.d.) is equally valuable as an example of an alternative approach to traditional practice in action. 
GRASAC. (n.d.) About GRASAC and the GKS. Great Lakes Research Alliance for the Study of Aboriginal Arts and Cultures.

LaFrance, A. (2016). The internet's favorite website. The Atlantic.

Marks, S. (2016). Program model: Local history Wikipedia edit-a-thon.

Mayer, A. (2016). Crowdsourcing, open data and precarious labour. Model View Culture 33.

McQuigge, M. (2013). Toronto professor learns not all editors are welcome on Wikipedia when class assignment backfires. The National Post.

Mills, A. (2016, June 2). Learning to Listen: Archival Sound Recordings and Indigenous Cultural Property. Paper presented at the Annual Conference of the Association of Canadian Archivists, Montreal, QC.

Mohyeddin, S. (2016). Editing the Wikipedia narrative of women in art. Torontoist.

Raub, C. (2015). Wikipedia, gender, and archives: Closing the gender gap on Wikipedia through archival outreach. Archival Outlook, May/June.

Reyes, S. (2014). Reliance on unpaid volunteers is turning public museums into a middle class commodity. New Statesman.

Simonite, T. (2013). The decline of Wikipedia. MIT Technology Review.

Torres, N. (2016). Why do so few women edit Wikipedia? Harvard Business Review.

Turner, J. and J. Schomberg (2016). Inclusivity, gestalt principles, and plain language in document design. In The Library with the Lead Pipe.

Wagner, C., Graells-Garrido, E., Garcia, D. \& Menczer, F. (2016). Women through the glass ceiling: gender asymmetries in Wikipedia. EPJ Data Science, 5.

Wiki Edu. (n.d.) For instructors.

Williams, S. (2016). Implications of archival labor. On Archivy.

Young, K. (2016, June 2). Community-Based Archives: their importance with the Canadian Context. Paper presented at the Annual Conference of the Association of Canadian Archivists, Montreal, QC.

Zandt, D. (2013). Yes, Wikipedia is sexist—that's why it needs you. Forbes. 